\title{
Isolated Renal Mucormycosis in Immunocompetent Children: A Report of Two Cases
}

\author{
Sai Saran, Kirti Naranje1, Mohan Gurjar, Dharmendra Bhadauria², Anupama Kaul2², Banani Poddar \\ Departments of Critical Care Medicine, ${ }^{1}$ Neonatology and ${ }^{2}$ Nephrology, Sanjay Gandhi Postgraduate Institute of Medical Sciences, Lucknow, Uttar Pradesh, India
}

\section{Abstract}

Isolated renal mucormycosis is a rare entity in children. It is potentially fatal when not detected and managed early with antifungal therapy, and surgery as and when needed. We present two immunocompetent children who developed this infection and subsequently succumbed to it. The diagnosis was established postmortem on renal biopsy specimens. We also discuss the 9 cases of isolated renal involvement in children published in literature.

Keywords: Mucormycosis, renal biopsy, zygomycosis

\section{INTRODUCTION}

Fungal infections are frequently missed and underdiagnosed in critically ill patients leading to significant morbidity and mortality. Among these, candidal infections predominate, followed by Aspergillus and Mucor. In a child with a history suggestive of urinary tract infection, features such as anuria and enlarged kidneys with the loss of vascularity on renal Doppler should alert the clinician and lead to further diagnostics. We present two apparently immunocompetent children who succumbed to a lethal fungal infection of the kidneys, mucormycosis, and suggest a clinical approach to manage such patients.

\section{Case Reports}

\section{Case 1}

A previously healthy 12-year-old boy presented with high fever, right flank pain, and oliguria of 5 days progressing to advanced renal failure. He underwent eight sessions of hemodialysis and received intravenous (IV) antibiotics. He continued to be febrile and anuric after 18 days when he developed respiratory distress and one episode of seizure and was referred to our hospital. On evaluation, the child (weight: $24 \mathrm{~kg}$ ) was in respiratory failure and shock, for which intubation, mechanical ventilation, and vasopressors were initiated and he was shifted to Intensive Care Unit (ICU). Workup revealed neutrophilic leukocytosis, deranged renal function, hyperkalemia,

\begin{tabular}{|l|l|}
\hline \multicolumn{3}{|c|}{ Access this article online } \\
\hline Quick Response Code: & Website: \\
\hline & www.ijccm.org \\
& \\
\hline
\end{tabular}

hyperlactatemia, and metabolic acidosis. He was started on antimicrobials (piperacillin-tazobactam, levofloxacin, and metronidazole), antiepileptic phenytoin, and continued on hemodialysis. Ultrasound imaging of the abdomen revealed hepatosplenomegaly, moderate ascites, and bilateral bulky kidneys with heterogeneous echotexture and a suspicious abscess in the lower pole of the left kidney. A computed tomography (CT) of the head was normal. Serology for $\mathrm{HIV}$, hepatitis B surface antigen ( $\mathrm{HBsAg}$ ), and hepatitis C virus $(\mathrm{HCV})$ was negative. Renal Doppler showed no flow in both main renal arteries and noncontrast $\mathrm{CT}$ of the kidneys showed multiple hypodensities in both kidneys [Figure 1a] though renal vessels could not be commented upon (IV contrast was not used in view of deranged renal function). With a suspicion of fungal pyelonephritis, the patient was started on conventional amphotericin B. On day 4 , he deteriorated further, and he succumbed to his illness on day 6 of his hospital stay. A postmortem kidney biopsy showed completely necrosed renal cortical parenchyma on light microscopy, with a few fungal elements with acute angle and broad-based hyphae, a picture suggestive of renal mucormycosis [Figure 1b].

Address for correspondence: Dr. Banani Poddar, Department of Critical Care Medicine, Sanjay Gandhi Postgraduate Institute of Medical Sciences, Lucknow - 226 014, Uttar Pradesh, India. E-mail: bananip@sgpgi.ac.in

This is an open access article distributed under the terms of the Creative Commons Attribution-NonCommercial-ShareAlike 3.0 License, which allows others to remix, tweak, and build upon the work non-commercially, as long as the author is credited and the new creations are licensed under the identical terms.

For reprints contact: reprints@medknow.com

How to cite this article: Saran S, Naranje K, Gurjar M, Bhadauria D, Kaul A, Poddar B. Isolated renal mucormycosis in immunocompetent children: A report of two cases. Indian J Crit Care Med 2017;21:457-9. 


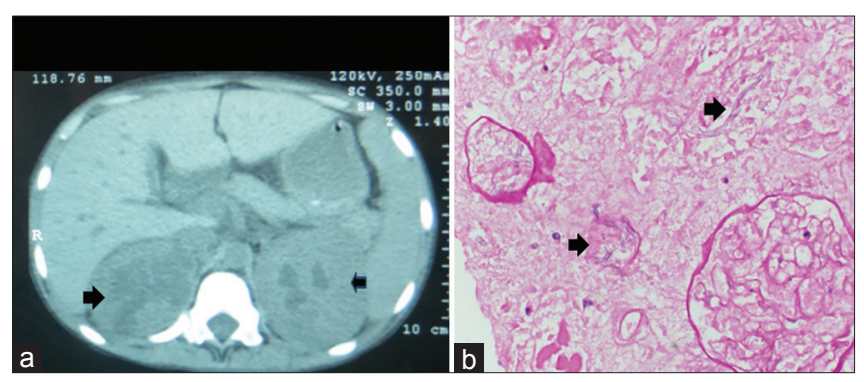

Figure 1: (a) Computed tomography abdomen showing hypodensities in both kidneys (b) postmortem renal biopsy showing aseptate fungal hyphae with acute angle branching (Case 1)

\section{Case 2}

A 10-year-old boy with no previous comorbidities, presented with high-grade fever and chills for 15 days, pain abdomen for 10 days; more on the left side and radiating to the back. A week later, he developed oliguria followed by anuria. There was no history of gross hematuria, altered sensorium, or seizures. He was treated at another hospital with peritoneal dialysis 3 days before referral to our center.

At admission, the child was pale, restless, and febrile with respiratory distress (weight: $30 \mathrm{~kg}$ ). He had abdominal distention and tenderness, more prominent in the left flank region, with free fluid. No organomegaly was noted. Investigations revealed anemia, neutrophilic leukocytosis, thrombocytopenia, and elevated procalcitonin. Blood biochemistry revealed raised urea nitrogen and creatinine, mild transaminitis, and deranged coagulation profile. Chest radiograph revealed bilateral pleural effusions and increased perihilar opacities suggestive of pulmonary edema. Abdominal ultrasound showed bilateral bulky kidneys with mild hydronephrosis and ascites. Workup for tropical diseases and immunological conditions was negative. Serology for HIV, HBsAg, and HCV was negative. The child was started on hemodialysis, empiric broad-spectrum antibiotics, and antifungal fluconazole along with supportive treatment. His clinical condition deteriorated with seizure and respiratory failure requiring invasive mechanical ventilation, and he was shifted to ICU. His subsequent course was complicated by septic shock requiring vasopressors and inotropes, severe metabolic acidosis with electrolyte disturbances requiring renal replacement therapy, and disseminated intravascular coagulation requiring transfusions of multiple blood products. Echocardiography revealed global hypokinesia with left ventricle ejection fraction of $20 \%$. The child succumbed to his illness on day 4 of admission. Blood cultures were sterile for bacteria and fungus. Postmortem kidney biopsy revealed acute necrosis of renal parenchyma with ghost cell outlines of glomeruli and tubules and fragments of aseptate fungal hyphae in the lumen of blood vessels and glomeruli suggestive of zygomycosis with infarct.

\section{DISCUSSION}

Mucormycosis is a rare fungal infection with high morbidity and mortality seen most often in immunocomprised hosts, especially in hematologic malignancies, transplant recipients, and diabetics. Other predisposing factors include IV drug abuse, deferoxamine therapy, burns, trauma, and malnutrition. Neutrophil or macrophage dysfunction, especially when associated with acidosis and hyperglycemia, predisposes to this infection. Isolated involvement of the kidneys by Mucorales is a rare entity in children. In a review of pediatric mucormycosis involving 187 patients, Roilides et al. described the most common patterns of organ involvement - rhinocerebral (18\%), cutaneous $(27 \%)$, pulmonary $(16 \%)$, and gastrointestinal $(21 \%) .{ }^{[1]}$ In 2004 , Jianhong et al. reported three cases with isolated renal involvement, of whom two were immunocompetent children, both of whom improved with surgery and antifungal therapy. ${ }^{[2]}$ More recently, Nayagam et al. reported an 18-month-old immunocompetent child with unilateral renal involvement, who improved after nephrectomy and had stable renal function. ${ }^{[3]}$ Previously described children with bilateral involvement [Table 1] have uniformly succumbed to their illness. The children described here are apparently immunocompetent and both developed bilateral pyelonephritis, secondary to mucormycosis, and worsened before any surgical intervention could be planned. To the best of our knowledge, these are the fourth and fifth cases of isolated renal mucormycosis in pediatric patients with bilateral involvement

Both the patients were nondiabetic, nonneutropenic, and not on any immunosuppressive medications; prolonged hospitalization with broad-spectrum antibiotic usage and hemodialysis could have placed them at risk for nosocomially acquired mucormycosis. However, community-acquired infections have been described. While relatively uncommon in the literature from developed countries, isolated renal involvement has been described by Indian authors. It is unknown as to what factors, inherited or environmental, place apparently immunocompetent individuals at risk of renal mucormycosis, in the absence of dissemination.

In a child with a history suggestive of urinary tract infection, features such as flank pain, gross hematuria or pyuria, acute kidney injury (anuria), and enlarged kidneys on ultrasonography, a high index of suspicion is required to suspect angioinvasive fungus. Further workup is warranted in such children with renal ultrasound to demonstrate hypoechoic shadows or hypodensities and Doppler to show altered blood flow in renal vessels. CT abdomen reveals enlarged kidneys with poor enhancement, hypodensities (parenchymal abscess), emphysematous changes, and infarcts (features of angioinvasion). Urine culture for fungus and kidney biopsy revealing broad-based (ribbon like) aseptate fungal hyphae branching at right angles clinches the diagnosis. Early aggressive management with amphotericin (or antifungal with activity against molds) is desirable and nephrectomy or partial excision to remove the infected and necrotic tissue is often required.

To summarize, isolated renal mucormycosis is a rare fungal infection with a fatal outcome unless detected early and treated aggressively with antifungal and surgery, if required. 


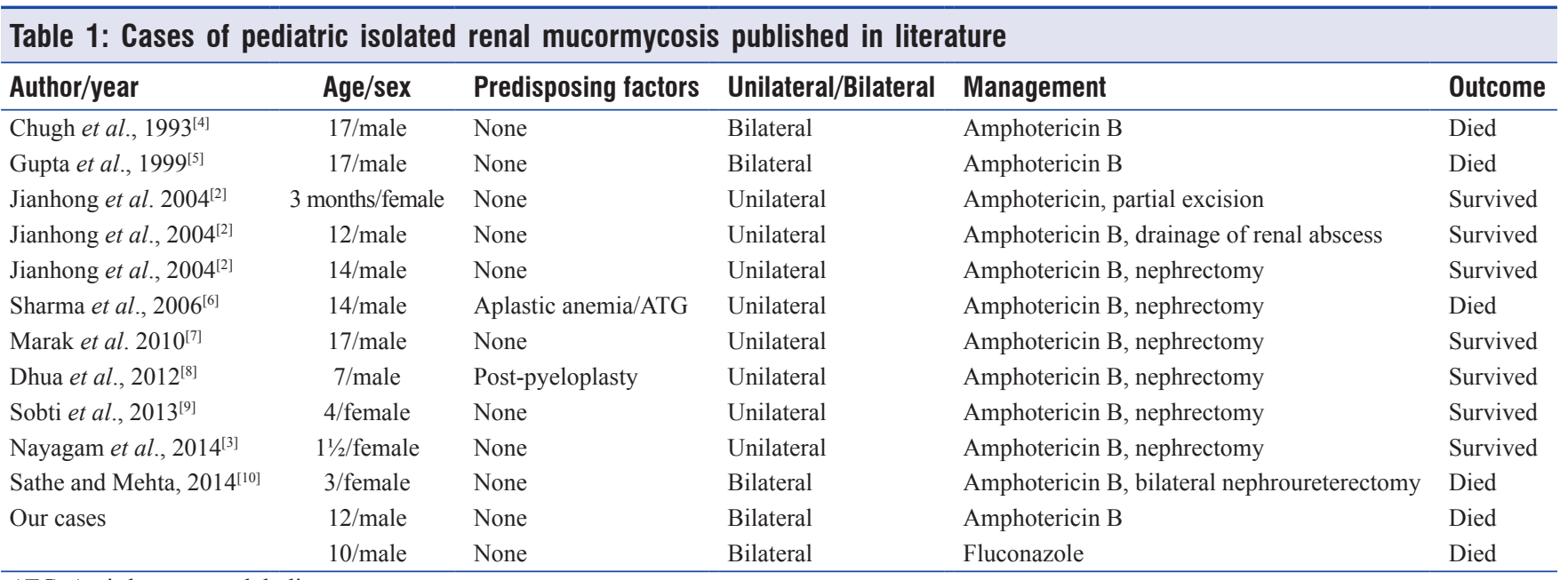

ATG: Anti-thymocyte globulin

\section{Acknowledgments}

We acknowledge the Department of Pathology for the photomicrographs of the renal biopsy.

\section{Financial support and sponsorship \\ Nil.}

\section{Conflicts of interest}

There are no conflicts of interest.

\section{References}

1. Roilides E, Zaoutis TE, Walsh TJ. Invasive zygomycosis in neonates and children. Clin Microbiol Infect 2009;15 Suppl 5:50-4.

2. Jianhong $\mathrm{L}$, Xianliang $\mathrm{H}$, Xuewu J. Isolated renal mucormycosis in children. J Urol 2004;171:387-8.

3. Nayagam LS, Vijayanand B, Balasubramanian S. Isolated renal mucormycosis in an immunocompetent child. Indian $\mathrm{J}$ Nephrol 2014;24:321-3
4. Chugh KS, Gupta KL, Sakhuja V. Renal mucormycosis: Computerized tomography findings and their diagnostic findings. Am J Kidney Dis 1993;22:393-7.

5. Gupta KL, Joshi K, Sud K, Kohli HS, Jha V, Radotra BD, et al. Renal zygomycosis: An under-diagnosed cause of acute renal failure. Nephrol Dial Transplant 1999;14:2720-5.

6. Sharma R, Shivanand G, Kumar R, Prem S, Kandpal H, Das CJ, et al. Isolated renal mucormycosis: An unusual cause of acute renal infarction in a boy with aplastic anaemia. Br J Radiol 2006;79:e19-21.

7. Marak RS, Misra R, Ansari MS, Dixit A, Poornima, Prasad KN, et al. Successful medical management of renal zygomycosis: A summary of two cases and a review of the Indian literature. Med Mycol 2010;48:1088-95

8. Dhua AK, Sinha S, Sarin YK, Khurana N. Isolated mucormycosis in a post-pyeloplasty kidney in an immuno-competent child. J Indian Assoc Pediatr Surg 2012;17:132-4.

9. Sobti P, Rakheja G, Mittal A. Isolated renal mucormycosis in a pediatric patient. J Case Rep 2013;3:390-2.

10. Sathe KP, Mehta KP. Irreversible fatal renal failure resulting from isolated renal mucormycosis. Saudi J Kidney Dis Transpl 2014;25:1312-4. 